\title{
Generalized uncertainty principle in Bianchi type I quantum cosmology
}

\author{
B. Vakili*and H. R. Sepangi ${ }^{\dagger}$ \\ Department of Physics, Shahid Beheshti University, Evin, Tehran 19839, Iran
}

October 25, 2018

\begin{abstract}
We study a quantum Bianchi type I model in which the dynamical variables of the corresponding minisuperspace obey the generalized Heisenberg algebra. Such a generalized uncertainty principle has its origin in the existence of a minimal length suggested by quantum gravity and sting theory. We present approximate analytical solutions to the corresponding Wheeler-DeWitt equation in the limit where the scale factor of the universe is small and compare the results with the standard commutative and noncommutative quantum cosmology. Similarities and differences of these solutions are also discussed.

PACS numbers: 04.60.-m, 04.60.Ds, 04.60.Kz
\end{abstract}

\section{Introduction}

A general predictions of any quantum theory of gravity is that there exists a minimal length below which no other length can be observed [1]-[6]. From perturbative string theory point of view $[1,2]$, such a minimal observable length is due to the fact that strings cannot probe distances smaller than the sting size. In the scale of this minimal size, the quantum effects of gravitation become as important as the electroweak and strong interactions. Thus, in the study of high energy physics phenomena such as the very early universe or in the strong gravitational fields of a black hole, one cannot neglect the effects of the existence of such a minimal length.

An important feature of the existence of a minimal length is the modification of the standard Heisenberg commutation relation in the usual quantum mechanics $[7,8]$. Such relations are known as the Generalized Uncertainty Principle (GUP). In one dimension, the simplest form of such relations can be written as

$$
\triangle p \triangle x \geq \frac{\hbar}{2}\left(1+\beta(\triangle p)^{2}+\gamma\right)
$$

where $\beta$ and $\gamma$ are positive and independent of $\triangle x$ and $\triangle p$, but may in general depend on the expectation values $\langle x\rangle$ and $\langle p\rangle$. The usual Heisenberg commutation relation can be recovered in the limit $\beta=\gamma=0$. As is clear from equation (1), this equation implies a minimum position uncertainty of $(\triangle x)_{\min }=\hbar \sqrt{\beta}$, and hence $\beta$ must be related to the Planck length. For a more general discussion on such deformed Heisenberg algebras, especially in three dimensions, see [9]. Now, it is possible to realize equation (1) from the following commutation relation between position and momentum operators

$$
[x, p]=i \hbar\left(1+\beta p^{2}\right)
$$

*email: b-vakili@sbu.ac.ir

†email: hr-sepangi@sbu.ac.ir 
where we take $\gamma=\beta\langle p\rangle^{2}$. More general cases of such commutation relations are studied in [7]-[11]. Also various applications of the low energy effects of the modified Heisenberg uncertainty relations have been extensively studied, see for example [12]-[15].

In this letter we consider a two dimensional minisuperspace of Bianchi type I cosmology in the GUP framework. We shall see that the corresponding Wheeler-DeWitt (WD) equation is a fourth order differential equation. Although, in general we cannot solve this equation exactly, we may obtain approximate analytical solutions in the limit of small scale factors i.e., in the region of the validity of GUP. We then compare the resulting wave functions with ordinary quantum cosmology and with noncommutative quantum cosmology where the latter is discussed in [16].

\section{The model}

Let us consider a cosmological model in which the spacetime is assumed to be of Bianchi type I whose metric can be written, working in units where $c=\hbar=16 \pi G=1$, as

$$
d s^{2}=-N^{2}(t) d t^{2}+e^{2 u(t)} e^{2 \beta_{i j}(t)} d x^{i} d x^{j},
$$

where $N(t)$ is the lapse function, $e^{u(t)}$ is the scale factor of the universe and $\beta_{i j}(t)$ determine the anisotropic parameters $v(t)$ and $w(t)$ as follows

$$
\beta_{i j}=\operatorname{diag}(v+\sqrt{3} w, v-\sqrt{3} w,-2 v) .
$$

To simplify the model we take $w=0$, which is equivalent to a universe with two scale factors in the form

$$
d s^{2}=-N^{2}(t) d t^{2}+a^{2}(t)\left(d x^{2}+d y^{2}\right)+c^{2}(t) d z^{2} .
$$

The anisotropy in the above metric can achieved by introducing a large scale homogeneous magnetic field in a flat FRW spacetime. Such a magnetic field results in a preferred direction in space along the direction of the field. If we introduce a magnetic field which has only a $z$ component, the resulting metric can be written in the form (5) where there are equal scale factors in the transverse directions $x$ and $y$ and a different one, $c(t)$, in the longitudinal direction $z$. The dynamics of such a universe is considered in [17].

Now, using the Einstein-Hilbert action

$$
\mathcal{S}=\int d^{4} x \sqrt{-g}(\mathcal{R}-\Lambda)
$$

where $g, \mathcal{R}$ and $\Lambda$ represent the determinant of the metric tensor, the scalar curvature and the cosmological constant respectively, we are led to the following Lagrangian in the minisuperspace $\{u, v\}$

$$
\mathcal{L}=\frac{6 e^{3 u}}{N}\left(-\dot{u}^{2}+\dot{v}^{2}\right)-\Lambda N e^{3 u} .
$$

Thus, with the choice of the harmonic time gauge $N=e^{3 u}[18]$, the Hamiltonian can be written as

$$
\mathcal{H}=\frac{1}{24}\left(-p_{u}^{2}+p_{v}^{2}\right)+\Lambda e^{6 u}
$$

Let us now proceed to quantize the model. In ordinary canonical quantum cosmology, use of the usual commutation relations $\left[x_{i}, p_{j}\right]=i \delta_{i j}$, results in the well known representation $p_{i}=-i \partial / \partial x_{i}$, from which the WD equation can be constructed. However, in the GUP framework, as was mentioned in the introduction, the existence of a minimal observable length requires new commutation relations between position and momentum operators. In more than one dimension a natural generalization of equation (2) is defined by the following commutation relations $[12,13,19]$

$$
\left[x_{i}, p_{j}\right]=i\left(\delta_{i j}+\beta \delta_{i j} p^{2}+\beta^{\prime} p_{i} p_{j}\right)
$$


where $p^{2}=\sum p_{i} p_{i}$ and $\beta, \beta^{\prime}>0$ are considered as small quantities of first order. Also, assuming that

$$
\left[p_{i}, p_{j}\right]=0
$$

the commutation relations for the coordinates are obtained as

$$
\left[x_{i}, x_{j}\right]=i \frac{\left(2 \beta-\beta^{\prime}\right)+\left(2 \beta+\beta^{\prime}\right) \beta p^{2}}{1+\beta p^{2}}\left(p_{i} x_{j}-p_{j} x_{i}\right) .
$$

As it is clear from the above expression, the coordinates do not commute. This means that to construct the Hilbert space representations, one cannot work in position space. It is therefore more convenient to work in momentum space, as is done in [12], [13] and [19]. However, since in quantum cosmology the wave function of the universe in momentum space has no suitable interpretation, we restrict ourselves to the special case $\beta^{\prime}=2 \beta$. As one can see immediately from equation (11), the coordinates commute to first order in $\beta$ and thus a coordinate representation can be defined. Now, it is easy to show that the following representation of the momentum operator in position space satisfies relations (9) and (10) (with $\beta^{\prime}=2 \beta$ ) to first order in $\beta$

$$
p_{i}=-i\left(1-\frac{\beta}{3} \frac{\partial^{2}}{\partial x_{i}^{2}}\right) \frac{\partial}{\partial x_{i}} .
$$

A comment on the above issue is that applying the GUP to a curved background such as a cosmological model needs some modifications [20]. Here, since we apply the GUP to the minisuperspace variables $u, v$ which correspond to a Minkowskian metric, we can safely use the above expressions without any modifications.

\section{Quantization of the model in the GUP framework}

Let us focus attention on the study of the quantum cosmology of the model described by the Hamiltonian (8). The corresponding commutation relations are as follows

$$
\begin{gathered}
{\left[u, p_{u}\right]=i\left(1+\beta p^{2}+2 \beta p_{u}^{2}\right), \quad\left[v, p_{v}\right]=i\left(1+\beta p^{2}+2 \beta p_{v}^{2}\right),} \\
{\left[u, p_{v}\right]=\left[v, p_{u}\right]=2 i \beta p_{u} p_{v},} \\
{\left[x_{i}, x_{j}\right]=\left[p_{i}, p_{j}\right]=0, \quad x_{i}(i=1,2)=u, v, \quad p_{i}(i=1,2)=p_{u}, p_{v} .}
\end{gathered}
$$

As we have mentioned in the previous section, in the special case when $\beta^{\prime}=2 \beta$, we have the following representations for $p_{u}$ and $p_{v}$ in the $u-v$ space which satisfy the commutation relations (13)-(15)

$$
p_{u}=-i\left(1-\frac{\beta}{3} \frac{\partial^{2}}{\partial u^{2}}\right) \frac{\partial}{\partial u}, \quad p_{v}=-i\left(1-\frac{\beta}{3} \frac{\partial^{2}}{\partial v^{2}}\right) \frac{\partial}{\partial v} .
$$

Now, using these representations for momenta in the Hamiltonian constraint (8), the WD equation can be written, up to first order in $\beta$, as

$$
\left\{\frac{\partial^{2}}{\partial u^{2}}-\frac{2}{3} \beta \frac{\partial^{4}}{\partial u^{4}}-\frac{\partial^{2}}{\partial v^{2}}+\frac{2}{3} \beta \frac{\partial^{4}}{\partial v^{4}}+24 \Lambda e^{6 u}\right\} \Psi(u, v)=0 .
$$

In the case of $\beta=0$, the ordinary quantum cosmology of the model can be recovered and its eigenfunctions can be written in terms of Bessel functions as follows [16]

$$
\Psi_{\nu}(u, v)=e^{-3 \nu v} J_{\nu}\left(2 \sqrt{\frac{2 \Lambda}{3}} e^{3 u}\right), \quad \Lambda>0
$$




$$
\Psi_{\nu}(u, v)=e^{3 i \nu v} K_{i \nu}\left(2 \sqrt{\frac{2|\Lambda|}{3}} e^{3 u}\right), \quad \Lambda<0 .
$$

The solutions of equation (17) are separable and may be written in the form $\Psi(u, v)=U(u) V(v)$, leading to

$$
\begin{gathered}
\frac{2}{3} \beta \frac{d^{4} V}{d v^{4}}-\frac{d^{2} V}{d v^{2}}+9 \nu^{2} V=0, \\
-\frac{2}{3} \beta \frac{d^{4} U}{d u^{4}}+\frac{d^{2} U}{d u^{2}}+\left(24 \Lambda e^{6 u}-9 \nu^{2}\right) U=0,
\end{gathered}
$$

where for having well-defined functions we use the positive separation constant $9 \nu^{2}$ in the case of a positive cosmological constant. Equation (20) is a fourth order linear differential equation whose solutions can be written in the form of exponential functions $e^{r v}$ where $r$ is the root of equation

$$
2 \beta r^{4}-3 r^{2}+27 \nu^{2}=0
$$

where

$$
r^{2}=\frac{3}{4 \beta}\left[1 \pm\left(1-24 \beta \nu^{2}\right)^{1 / 2}\right] .
$$

To achieve the correct limit for $\beta \rightarrow 0$, we take the negative sign in the above expression. Thus, up to first order in $\beta$, the solution of equation (20) reads

$$
V(v)=e^{-3 \nu\left(1+3 \beta \nu^{2}\right) v} .
$$

Now, let us deal with equation (21). Taking $\beta=0$ in this equation yields the ordinary WD equation where its solutions in the case of a positive $\Lambda$ are given by equation (18). In the case when $\beta \neq 0$, we cannot solve equation (21) exactly, but we can provide an approximate method whose domain of validity is given by the solution of a second order differential equation. To this end, we note that the effects of $\beta$ become important at the Planck scale, or in cosmology language in the very early universe, that is, when the scale factor is small, $e^{3 u} \rightarrow 0$. Thus, if we substitute solution (18) in the $\beta$-term of equation (21), we may obtain approximate analytical solutions in the region $e^{3 u} \rightarrow 0$. The limiting behavior of solution (18) in the region $e^{3 u} \rightarrow 0$ is [21]

$$
J_{\nu}\left(2 \sqrt{\frac{2 \Lambda}{3}} e^{3 u}\right) \rightarrow \frac{1}{\Gamma(\nu+1)}\left(\sqrt{\frac{2 \Lambda}{3}}\right)^{\nu} e^{3 \nu u},
$$

and therefore its fourth derivative is

$$
\frac{d^{4} U}{d u^{4}}=(3 \nu)^{4} U
$$

Substituting this result into equation (21) leads to the following equation

$$
\frac{d^{2} U}{d u^{2}}+\left[24 \Lambda e^{6 u}-9\left(\nu^{2}+6 \beta \nu^{4}\right)\right] U=0,
$$

with solution, up to first order in $\beta$, as

$$
U(u)=J_{\nu\left(1+3 \beta \nu^{2}\right)}\left(2 \sqrt{\frac{2 \Lambda}{3}} e^{3 u}\right) .
$$

For a negative cosmological constant, the above procedure leads to the solutions

$$
e^{3 i \nu\left(1-3 \beta \nu^{2}\right) v}
$$



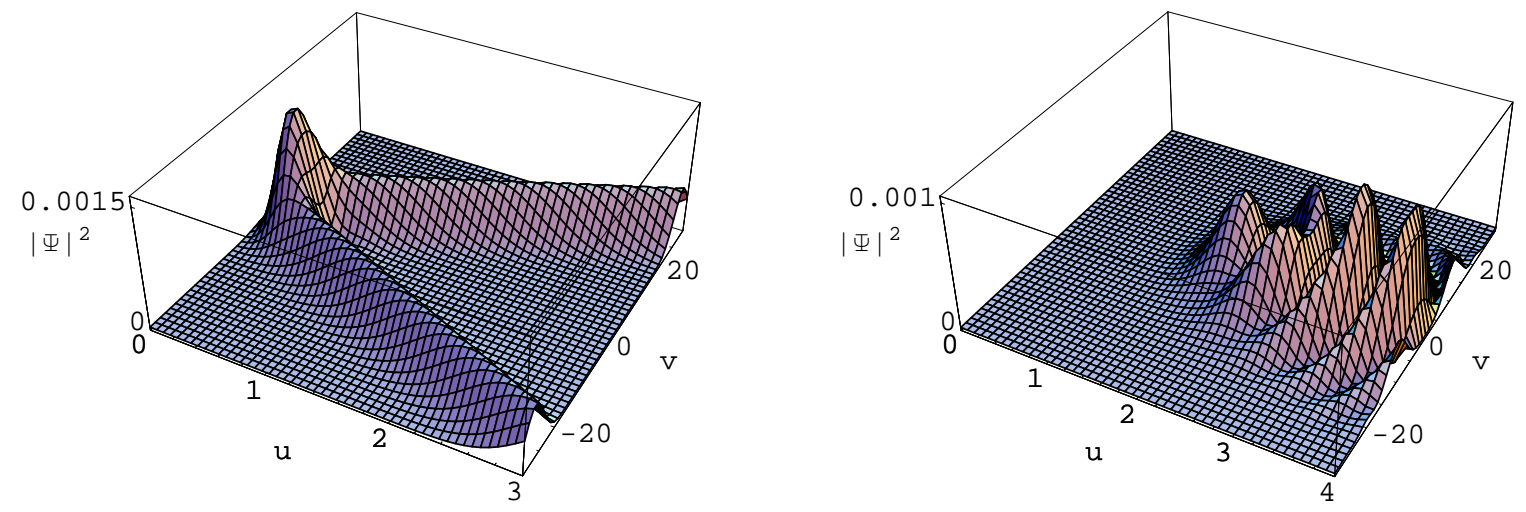

Figure 1: The figure on the left shows the square of the commutative wave function while the figure on the right, the square of the noncommutative wave function. The figures are plotted for a negative cosmological constant.

for $V(v)$ and modified Bessel function

$$
K_{i \nu\left(1-3 \beta \nu^{2}\right)}\left(2 \sqrt{\frac{2 \Lambda}{3}} e^{3 u}\right),
$$

for $U(u)$. Thus, the eigenfunctions of the WD equation can be written as

$$
\begin{array}{cc}
\Psi_{\nu}(u, v)=e^{-3 \nu\left(1+3 \beta \nu^{2}\right) v} J_{\nu\left(1+3 \beta \nu^{2}\right)}\left(2 \sqrt{\frac{2 \Lambda}{3}} e^{3 u}\right), & \Lambda>0, \\
\Psi_{\nu}(u, v)=e^{3 i \nu\left(1-3 \beta \nu^{2}\right) v} K_{i \nu\left(1-3 \beta \nu^{2}\right)}\left(2 \sqrt{\frac{2|\Lambda|}{3}} e^{3 u}\right), \quad \Lambda<0 .
\end{array}
$$

We may now write the general solutions to the WD equation as a superposition of the eigenfunctions

$$
\Psi(u, v)=\int_{-\infty}^{+\infty} C(\nu) \Psi_{\nu}(u, v) d \nu
$$

where $C(\nu)$ can be chosen as a shifted Gaussian weight function $e^{-a(\nu-b)^{2}}$.

\section{Comparison of the results}

In a previous work [16], we obtained the solutions of the same problem in a noncommutative phase space with the following commutation relations

$$
\left[u_{n c}, v_{n c}\right]=i \theta, \quad\left[u_{n c}, p_{u}\right]=\left[v_{n c}, p_{v}\right]=i, \quad\left[p_{u}, p_{v}\right]=0
$$

and showed that the eigenfunctions of the corresponding WD equation in such a space are given by

$$
\begin{array}{cc}
\Psi_{\nu}(u, v)=e^{-3 \nu v} J_{\nu}\left(2 \sqrt{\frac{2 \Lambda}{3}} e^{3\left(u-\frac{3}{2} \nu \theta\right)}\right), \quad \Lambda>0, \\
\Psi_{\nu}(u, v)=e^{3 i \nu v} K_{i \nu}\left(2 \sqrt{\frac{2|\Lambda|}{3}} e^{3\left(u-\frac{3}{2} \nu \theta\right)}\right), \quad \Lambda<0 .
\end{array}
$$




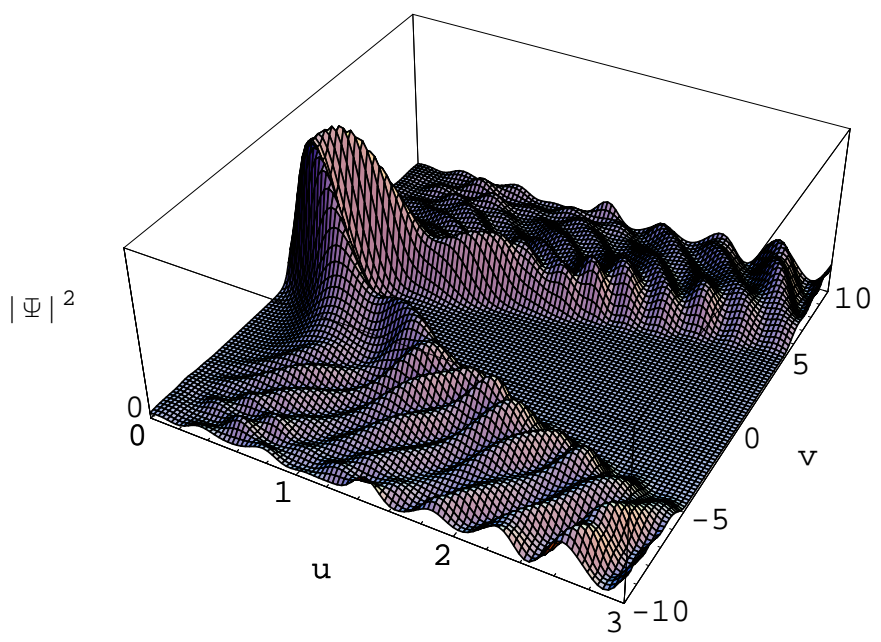

Figure 2: The square of wave function in the GUP framework. The figure is plotted for a negative cosmological constant.

As can be seen, the general solutions are again in the form of an expression like (28). Although in the present study and with the choice $\beta^{\prime}=2 \beta$, the phase space variables $u, v$ commute with each other, in general equation (11) shows that GUP leads naturally to a noncommutative generalization of position space which may point to a close relationship between noncommutativity and GUP. The study of such phenomena as IR/UV mixing and non-locality, Lorentz violation and new physics at very short distances in noncommutative [22]-[26] and GUP [27, 28] frameworks, would pave the way for a more clear understanding of this relationship.

Figures 1 and 2 show the square of wave functions in the context of an ordinary commutative phase space, noncommutative phase space and when the phase space variables obey the GUP relations. As is clear from these figures, in the ordinary commutative case $(\theta=\beta=0)$, we have only one possible universe around a nonzero value of $u$ and $v=0$, which means that the universe in this case approaches a flat FRW one. On the other hand we see that noncommutativity causes a shift in the minimum value of $u$ corresponding to the spatial volume. The emergence of new peaks in the noncommutative wave packet may be interpreted as a representation of different quantum states that may communicate with each other through tunnelling. This means that there are different possible universes (states) from which our present universe could have evolved and tunnelled, from one state to another (see also [29]). Such behavior also occurs in figure 2 which shows the square of the GUP wave function, showing that from the point of view adopted here, noncommutativity and GUP may be considered as similar concepts. However, there is an important difference, namely, that the noncommutative wave function not only peaks around $v=0$, but appear symmetrically around a nonzero value of $v$, which is the characteristic of an anisotropic universe. On the other hand, the GUP wave function as is seen in the figure, has many peaks around the value $v=0$ and therefore from the point of predicting an isotropic universe the GUP wave packet behaves like the ordinary commutative case.

\section{Conclusions}

In this paper we have studied the effects of the existence of a minimal length scale on the quantum states of a Bianchi type I cosmology. This phenomena yields a deformed Heisenberg commutation relation between the position and momentum operators which are known as the GUP. Although in more than one dimension, because of the existence of a noncommutative relation between the space 
operators it is not possible to represent the momentum operator in the position space, in the special case when the GUP parameters obey the relation $\beta^{\prime}=2 \beta$, the space operators commute and a coordinate representation of momenta can be defined. Since a wave function describing the quantum state of the universe does not not have a suitable interpretation in momentum space, working in the above special case is crucial in our work.

When the phase space variables obey the ordinary commutation relations we have seen that there is only one possible isotropic universe. Upon considering the GUP and through finding the approximate analytical solutions of the WD equation in the limit of small scale factors, we have studied the corresponding quantum cosmology and seen that in the presence of the GUP, the square of the wave function of the universe has several peaks. This behavior, also occurring in the noncommutative quantum model studied in a previous work [16], may be related to different states in the early universe from which our present universe could have evolved. Although, both noncommutativity and GUP predict many possible initial universes, this is not the case in predicting the isotropicity. In the case of a noncommutative cosmology the universe behaves anisotropicaly while the GUP predicts an isotropic universe.

\section{References}

[1] D. J. Gross and P. F. Mende, Nucl. Phys. B 303, (1988) 407.

[2] D. Amati, M. Ciafaloni and G. Veneziano, Phys. Lett. B 216 (1989) 41.

[3] M. Kato, Phys. Lett. B 245 (1990) 43.

[4] S. Haro, J. High Energy Phys. JHEP 10 (1998) 023.

[5] L. G. Garay, Int. J. Mod. Phys. A 10 (1995) 145, (gr-qc/9403008).

[6] K. Konishi, G. Paffuti and P. Provero, Phys. Lett. B 234 (1990) 276.

[7] A. Kempf, G. Mangano and R. B. Mann, Phys. Rev. D 52 (1995) 1108, (hep-th/9412167).

[8] A. Kempf and G. Mangano, Phys. Rev. D 55 (1997) 7909, (hep-th/9612084).

[9] M. Maggiore, Phys. Lett. B 319 (1993) 83,

M. Maggiore, Phys. Rev. D 49 (1994) 5182,

A. Kempf, J. Math. Phys. 35 (1994) 4483,

R. J. Adler and D. I. Santiago, Mod. Phys. Lett. A 14 (1999) 1371.

[10] A. Kempf, Phys. Rev. D 63 (2001) 083514,

A. Kempf and J. C. Niemeyer, Phys. Rev. D 64 (2001) 103501,

L. N. Chang, D. Minic, N. Okamura and T. Takeuchi, Phys. Rev. D 65 (2002) 125027.

[11] H. Hinvichsen and A. Kempf, J. Math. Phys. 37 (1996) 2121.

[12] F. Brau, J. Phys. A 32 (1999) 7691, (quant-ph/9905033).

[13] R. Akhoury and Y. P. Yao, Phys. Lett. B 572 (2003), (hep-ph/0302108).

[14] S. Hossenfelder, M. Bleicher, S. Hofmann, J. Ruppert, S. Scherer and H. Stöcker, Phys. Lett. B 575 (2003) 85, (hep-th/0305262).

[15] K. Nozari and T. Azizi, Gen. Rel. Grav. 38 (2006) 735.

[16] B. Vakili, N. Khosravi and H. R. Sepangi, Class. Quantum Grav. 24 (2007) 931, (gr-qc/0701075).

[17] E. J. King and P. Coles, Class. Quantum Grav. 24 (2007) 2061. 
[18] U. Bleyer, V. D. Ivashchuk, V. N. Melnikov and A. Zhuk, Nucl. Phys. B 429 (1994) 177, (grqc/9405020),

V. D. Ivashchuk, V. N. Melnikov and A. I. Zhuk, Nuov. Cim. D 104 , (1989) 575.

[19] A. Kempf, J. Phys. A 30 (1997) 2093 (hep-th/9604045),

I. Dadic, L. Jonke and S. Meljanac, Phys. Rev. D 67 (2003) 087701, (hep-th/0210264).

[20] S. F. Hassan and M. S. Sloth, Nucl. Phys. B 674 (2003) 434,

M. V. Battisti and G. Montani, The Big-Bang Singularity in the framework of a Generalized Uncertainty Principle, (gr-qc/0703025),

F. Scardigli, Phys. Lett B 452 (1999) 39, (hep-th/9904025),

F. Scardigli and R. Casadio, Class. Quantum Grav. 20 (2003) 3915.

[21] W. Magnus, F. Oberhettinger and R. P. Soni, Formulas and Theorems for the Special Functions of Mathematical Physics, Springer-Verlag, Berlin, 1966.

[22] S. Minwalla, M. Van Raamsdonk and N. Seiberg, J. High Energy Phys. JHEP 02 (2000) 020, (hep-th/9912072).

[23] S. M. Carroll, J. A. Harvey, V. A. Kostelesky, C. D. Lane and T. Okamoto, Phys. Rev. Lett. 87 (2001) 141601, (hep-th/0105082),

C. E. Carlson, C. D. Carone and R. F. Lebed, Phys. Lett. B 518 (2001) 201, (hep-ph/0107291),

C. E. Carlson, C. D. Carone and R. F. Lebed, Phys. Lett. B 549 (2002) 337, (hep-ph/0209077).

[24] R. J. Szabo, Phys. Rep. 387 (2003) 207, (hep-th/0109162).

[25] M. R. Douglas and N. A. Nekrasov, Rev. Mod. Phys. 73 (2002) 977, (hep-th/0106048).

[26] I. Bars, Nonpertubative Effects of Extreme Localization in Noncommutative Geometry , (hepth/0109132).

[27] A. W. Peet and J. Polchinski, Phys. Rev. D 59 (1999) 065011.

[28] C. M. Hull, J. High Energy Phys. JHEP 07 (1998) 021, V. Balasubramanian, P. Horava and D. Minic, J. High Energy Phys. JHEP 05 (2001) 043, V. Balasubramanian, J. de Boer and D. Minic, Phys. Rev. D 65 (2002) 123508.

[29] H. Garcia- Compean, O. Obregon and C. Ramirez, Phys. Rev. Lett. 88 (2002) 161301, (hepth/0107250). 